\title{
Aa. Vv., Lecteurs et collectionneurs de textes clandestins à l'âge classique
}

\section{Luigi Luison}

\section{Q OpenEdition}

1 Journals

\section{Edizione digitale}

URL: https://journals.openedition.org/studifrancesi/26982

DOI: 10.4000/studifrancesi.26982

ISSN: 2421-5856

\section{Editore}

Rosenberg \& Sellier

\section{Edizione cartacea}

Data di pubblicazione: 1 avril 2007

Paginazione: 227-228

ISSN: 0039-2944

\section{Notizia bibliografica digitale}

Luigi Luison, «Aa. VV., Lecteurs et collectionneurs de textes clandestins à l'âge classique», Studi Francesi [Online], 151 (LI | I) | 2007, online dal 30 novembre 2015, consultato il 23 novembre 2021. URL: http:// journals.openedition.org/studifrancesi/26982 ; DOI: https://doi.org/10.4000/studifrancesi.26982

Questo documento è stato generato automaticamente il 23 novembre 2021.

\section{(c) (i) (9)}

Studi Francesi è distribuita con Licenza Creative Commons Attribuzione - Non commerciale - Non opere derivate 4.0 Internazionale. 


\title{
Aa. Vv., Lecteurs et collectionneurs de textes clandestins à l'âge classique
}

\author{
Luigi Luison
}

\section{NOTIZIA}

Aa. Vv., Lecteurs et collectionneurs de textes clandestins à l'âge classique, «La Lettre Clandestine», n. 12, maggio 2004, pp. 498.

1 Questo numero della rivista, pubblicata in collaborazione con il C.N.R.S. dalle Università di Saint-Étienne, Paris I, Paris IV e Paris XII, presenta, nella prima parte del volume, un ampio dossier tematico intitolato "Lecteurs et collectionneurs de textes clandestins à l'âge classique". Come reperire e definire un lettore, un collezionista? Che cosa contraddistingue un copista da un autore? E soprattutto, come utilizzare le loro testimonianze? Questi sono solo alcuni dei quesiti a cui gli autori dei saggi qui esposti cercano di dare delle risposte.

2 Éric PUISAIS (Sur la trace de quelques manuscrits clandestins au travers des Remarques en lisant du marquis René Louis d'Argenson, pp. 47-57) presenta alcuni percorsi di ricerca a partire da un fondo di archivio (che va dal 1740 alla fine del 1800) ancora misconosciuto e depositato dalla famiglia d'Argenson alla Biblioteca Universitaria di Poitiers nel 1974, in cui l'A. ha rintracciato alcuni manoscritti filosofici clandestini del Settecento, soprattutto grazie ad un'attenta analisi della copia manoscritta delle Remarques en lisant di René Louis d'Argenson.

3 Catherine HÉMON-FABRE et Alain мотноU (Un lecteur des curés Guillaume et Meslier, le chavalier de La Vieuville, pp. 59-96). Nel 1752 Charles-Louis-Marie de la Vieuville redige le sue memorie, il cui testo è conservato, oggi, in un fondo privato che contiene altri documenti provenienti dalla medesima famiglia. Questi Mémoires contengono testimonianze importanti su due letture "clandestine" effettuate da Vieuville: una riguarda il Traité des trois imposteurs del curato Guillaume, l'altra il Mémoire des pensées et 
sentiments del curato Meslier. Il saggio degli Autori riporta, oltre all'analisi dei Mémoires di Charles-Louis-Marie de la Vieuville, un estratto di queste due testimonianze.

Antony MCKENNA (Le marquis d'Argens et les manuscrits clandestins, pp. 97-120) organizza il suo campo di indagine su un manoscritto clandestino molto conosciuto, Doutes sur la religion dont on cherche l'éclarcissement de bonne foi, che il marchese d'Argens cita nei suoi Mémoires del 1735 a proposito delle sue perplessità sulla religione cristiana. Il percorso dell'A. si precisa attraverso l'analisi di altre letture filosofiche del marchese.

5 Maria Susana SEGUIN (Boulanger lecteur de manuscrits philosophiques clandestins?, pp. 121-134). L'opera di Boulanger, che conferma la sua profonda conoscenza dei grandi scrittori dell'Antichità, ha esercitato una certa influenza su tutta una generazione di pensatori: coloro che da una parte si interessavano alle origini della società e coloro che dall'altra si impegnavano attivamente nel dibattito religioso. Maria Susana Seguin si interessa alle lacune significative presenti nelle fonti manoscritte citate da Boulanger e che sono alla base del suo pensiero, per meglio definire la relazione esistente tra questo pensatore ed i manoscritti filosofici, nonché di evidenziare i punti di incontro più significativi con i testi polemici del Settecento.

6 Olivier вцосн (Lettres à Sophie et manuscrits clandestins: des détails troublants, pp. 135-145). In questo lavoro l'A. mette l'accento sulla relazione che intercorre tra la versione stampata delle Lettres à Sophie, un testo clandestino anonimo, e il manoscritto (Mazarine 1183) Lettres sur la religion, sur l'âme humaine, et sur l'existence de Dieu. Rispetto alla versione manoscritta, quella stampata pone ancora, secondo l'A., una serie di problematiche parzialmente irrisolte, sebbene il rapporto tra $i$ due testi sembri condurci sulle tracce di letture di manoscritti clandestini.

7 Antony mCKenNa (La bibliothèque de l'abbé Sépher, pp. 147-158) presenta brevemente la biblioteca dell'abate Sépher che oggi è parzialmente conosciuta per via del catalogo dei suoi libri e di qualche manoscritto sparso tra le biblioteche municipali di Aix-enProvence, Rouen e Saint-Pétersbourg. Si tratta di un biblioteca che non esiste più in quanto tale, ma che l'A. ricostruisce per cogliere l'ardore bibliofilo e le preferenze molto specialistiche del collezionista. Attraverso questa ricostruzione l'A. evidenzia la presenza di manoscritti filosofici clandestini copiati e annotati per mano di Sépher e che facevano parte della sua biblioteca personale.

8 Laurence MACÉ (De la bibliothèque au journal intime: Giuseppe Pelli collectionneur et lecteur, pp. 159-175) si occupa della diffusione delle idee e dei testi filosofici intorno al 1760. Questa fase di propaganda vede la clandestinità investire in maniera massiccia la sfera della stampa e si ridefinisce in generi e forme del tutto nuovi. In questa prospettiva, l'A. cerca di rispondere ad alcuni interrogativi sorti in fase di ricostruzione di tale processo, tracciando il profilo di Giuseppe Pelli Bencivenni, funzionario fiorentino della seconda metà del Settecento, collezionista e lettore che ha costituito in Italia una collezione di opere clandestine in lingua francese.

9 Christian DEL VENTO (Lumières et littérature clandestine dans la bibliothèque d'un collectionneur italien à Paris: le cas de Vittorio Alfieri, pp. 177-200). Tra gli autori italiani vissuti a Parigi alla fine del Settecento, spicca la figura di Vittorio Alfieri. Ciò che attira l'attenzione dell'A. è la storia complessa delle biblioteche che Alfieri costituì nel capoluogo francese. Dalla ricostruzione del catalogo della Biblioteca Nazionale Centrale di Firenze, emerge la presenza di testi classici antichi e italiani. Nonostante non ci siano tracce, nella prima biblioteca del poeta, della presenza di testi clandestini, l'A. ritiene che Alfieri abbia avuto accesso ai classici della letteratura clandestina fin dalla 
giovinezza e che tra i 500 volumi non recensiti dalle autorità rivoluzionarie incaricate della loro confisca, ci siano stati testi clandestini, manoscritti o a stampa. Tuttavia, il campo è ancora aperto.

Dominique VARRY (Jean-Baptiste Reguilliat, imprimeur-libraire lyonnais destitué en 1767, pp. 201-218). Chi era Jean-Baptiste Reguilliat e perchè si attirò l'ostilità delle autorità? Cercando una risposta a queste domande, l'A. traccia il ritratto di questo libraiostampatore, colto in flagrante nel 1762 durante la stampa del Contrat social che gli valse la carcerazione nella prigione di Pierre-Scize. Secondo l'A., il caso Reguilliat è emblematico della situazione lionese della seconda metà del Settecento e induce a ritenere che molti alti librai-stampatori, oggi totalmente sconosciuti, abbiano operato al fianco di Reguilliat o, quantomeno, sulle sue tracce.

Questa prima sezione si chiude con le considerazioni di Geneviève ARTIGAS-MENANT che fornisce alcuni strumenti di lavoro al fine di evitare di ridurre il mondo dei lettori e dei collezionisti di testi clandestini ai soli casi documentati. È importante procedere, secondo l'A., non solo attraverso un esame metodico dei cataloghi delle biblioteche, alla ricerca di indizi e piste diverse, ma soprattutto avvicinarsi al mondo dei lettori e dei collezionisti di testi clandestini studiando i loro fornitori, come nel caso di Reguilliat di Lione.

12 La seconda parte, intitolata "Varia", espone i lavori che non sono stati presentati alla giornata-studio tenutasi a Créteil nel giugno 2003 e nell'agosto dello stesso anno all'undicesimo congresso internazionale dei Lumi di Los Angeles.

13 Il primo dei lavori presentati è il saggio di Jean-Pierre CAVAILlé (Contre la censure des livres. Deux textes extraits d'oeuvre italiennes traduites en 1644: Le Courrier dévalisé de Ferrante Palavicino - Lettre contre ceux qui défendent les livres - et le Divorce céleste, pp. 225-236) che ci presenta un estratto in originale delle due opere citate. Le due opere, pubblicate clandestinamente nel 1643-1644, furono oggetto di una traduzione francese pubblicata quasi contemporaneamente a Venezia e destinata ad un pubblico transalpino. Questo fenomeno di traduzioni attesta una forte circolazione di letteratura libellista a carattere eterodosso, persino libertino, tra Venezia e la Francia nella metà del Seicento.

Daniel LACERDA (La pression du pouvoir inquisitorial contre la formation de bibliothèques au Portugal: 1640-1660, pp. 237-272) racconta, attraverso lo scambio epistolare tra D. Vicente Nogueira marchese di Nisa e il re Jean IV, le peripezie di una vera tragedia che privò il Portogallo dell'accesso alla più avanzata documentazione e all'evoluzione spirituale e scientifica, in un'epoca decisiva per lo sviluppo delle idee e del potere di Stato.

Gianluca MоRI e Alain мотноu (L'âme matérielle; De la conduite qu'un honnête homme doit garder pendant sa vie; Préface du traité sur la religion de M.**. Trois manuscrits, un seul auteur?, pp. 311-339). Riallacciandosi al saggio incluso nella prima parte del volume, l'A. fa notare come i Mémoires del conte di La Vieuville apporti nuovi elementi sulle attività clandestine del curato di Fresnes, Étienne Guillaume, e contemporaneo di Jean Meslier. Questa testimonianza porta gli A. a riesaminare lo statuto di un manoscritto della Biblioteca dell'Arsenale, Préface du traité sur la religion de M.***, identificato nel 1993 come una raccolta di frammenti del trattato perduto di Guillaume, e ad interessarsi al rapporto tra questa Préface e altri due trattati manoscritti clandestini più noti, L'âme matérielle e De la conduite qu'un honnête homme doit garder pendant sa vie. Del trattato perduto, gli A. hanno trovato tracce anche in una lettera che Voltaire indirizzò 
a d'Alembert il 9 gennaio 1773, come testimonia il loro saggio successivo (Voltaire, Guillaume et la Priore du curé de Fresnes, pp. 365-379).

Nel lavoro che segue, gli stessi Gianluca Mori e Alain Mothou (Des Miracles, Des Oracles, Des Démons. Trois autres fragments du traité perdu du curé Guillaume?, pp. 341-363) focalizzano l'attenzione su altri tre testi che figurano nella biblioteca del curato Guillaume: Des Miracles e Des Oracles, due trattati manoscritti e stampati nel 1775 con il titolo La Fausseté des miracles, e un capitolo sull'origine della credenza nei demoni che figura nel testo stampato.

17 Miguel BENítez (Nouvelles d'un nouveau monde: Sur l'origine marine de l'homme dans Telliamed, pp. 273-295) lavora sul trattato di Benoît de Maillet, Telliamed, sull'origine marina dell'uomo, che conobbe nel Settecento un grande successo.

18 Madeleine ALCOVER (Sésame, ouvre-toi! Les trésors cachés du Minutier Central, pp. 297-309) espone brevemente le difficoltà che si incontrano nel momento in cui si affrontato ricerche di documenti notarili. Nel caso specifico, l'A. si riferisce al ritrovamento di due minute notarili del 1578, scoperte ad Auxerre e nelle quali compare il nome di un noto nobile parigino: Savinien Cirano.

Questo numero della rivista si conclude con numerosi comptes rendus e con un Bulletin d'Information. 